\title{
Potentiale und Grenzen umweltbasierter Konflikttransformation: Erkenntnisse aus dem Arava Institute for Environmental Studies in Israel
}

\author{
N. Fricke, P. Rodrian, and H.-M. Zademach \\ KU Eichstätt-Ingolstadt, Germany \\ Correspondence to: P. Rodrian (mga303@ku.de)
}

Received: 12 November 2015 - Revised: 17 March 2016 - Accepted: 21 March 2016 - Published: 7 June 2016

\begin{abstract}
Kurzfassung. Der Beitrag untersucht am Beispiel des Arava Institute for Environmental Studies in Israel, wie Friedensbildungsmaßnahmen mit Bezug zu Umweltthemen die Sichtweisen auf einen Konflikt bei Akteuren auf Graswurzelebene verändern können. Ausgangsüberlegung ist dabei, Umwelt nicht nur als Teil des Konfliktes zu interpretieren, sondern als konfliktneutralen Katalysator einzusetzen. Die auf dieser Basis erreichte Veränderung der Wahrnehmung des Konflikts wird in Form von konstruierten Raumbildern konzeptualisiert. Die empirischen Einblicke zeigen zum einen die Bedeutung von physischen wie sozialen Orten und Räumen als Rahmen für die konstruktive Auseinandersetzung. Zum anderen lässt sich in Bezug auf die Konfliktwahrnehmung ein Bruch zwischen einer positiven Veränderung des Bildes der anderen Gruppe und der persistenten Konstruktion des Erbes ableiten.
\end{abstract}

1

„Arava [Institute for Environmental Studies, Israel] definitely changed my way of thinking!“... „Has it also changed your behavior? Is there anything you do different, since you are an Arava Alumni?““ ..., ,No, actually not!““

Vor dem Hintergrund aktueller kriegerischer Auseinandersetzungen und der daraus resultierenden „Flüchtlingskrise“ stellt die Frage nach der Transformation von Konflikten ein interdisziplinäres Forschungsfeld dar, dem sich auch Geographinnen und Geographen vermehrt widmen. Für die Transformation von Konflikten ist Bildung ein Schlüssel (Lenhart et al., 2007). Dazu zählen auch Bildungsinitiativen, die auf Track 3 Ebene - also auf Ebene von möglichen Multiplikatoren aus der Zivilgesellschaft - versuchen, die Sichtweisen von beteiligten Akteuren eines Konfliktes hin zu einem positiven Frieden zu verändern (weiterführend zu Multi-Track z.B. Jäger, 2010). Solchen friedensbildenden Maßnahmen wird eine entscheidende Bedeutung für die Veränderung von tief verwurzelten Konflikten zugesprochen (Salomon, 2013; Davies, 2010; Bush und Salterelli, 2000). In der Literatur werden entsprechende Ansätze unter der zivilgesellschaftli- chen Strategie von Peace Building gefasst (Lederach, 1995, 1997; parallel werden Sicherheits-, Liberalisierungs- und Institutionalisierungsstrategien diskutiert, z.B. Marten, 2004; Collier, 2003; Fukuyama, 2004). Ziel der zivilgesellschaftlichen Konflikttransformation ist die Schaffung einer Basis für Versöhnung und Koexistenz in der Bevölkerung, indem die Sichtweisen von Individuen bezüglich der Interpretation von (oft instrumentalisierten) gesellschaftlichen und konfliktbezogenen Konstruktionen verändert und dadurch Multiplikatoren angestoßen werden. Auch wenn die Wirksamkeit diese Strategie kurzfristig auf dem Makro-Level, d.h. auf politischer bzw. Track 1 Ebene, kaum sichtbar ist, hat sie mittelbis langfristig unverzichtbaren Einfluss auf sozialen Wandel und damit die Transformation von Konflikten (Jäger, 2010; Salomon und Crains, 2010).

Im Zentrum der zivilgesellschaftlichen Konflikttransformation steht die begleitete Zusammenführung von Konfliktgruppen, um Differenzen zu überwinden und einen aktiven Beitrag zur Vertrauensbildung zu leisten (Jäger, 2010; Stürmer, 2008; Dollase, 2001; Jonas, 1998). Dieses Vorgehen, auch als Kontakthypothese bezeichnet, ist nicht unumstritten, da auch negative Entwicklungen durch den Kontakt der Konfliktparteien festgestellt werden konnten (Brown, 2002). 
Über den einfachen Kontakt hinaus gehen Beziehungen und Netzwerke, die durch friedensbildende Track 3 Maßnahmen aufgebaut werden. In diesem Zusammenhang beschreibt Putnam (2000) zwei unterschiedliche Phänomene, bonding und bridging. Innerhalb von Gruppen, die an einer Friedensbildungsmaßnahme teilnehmen, können ein verbessertes Vertrauensverhältnis und eine gemeinsame Identität entstehen (bonding). Wenn indes die Gruppe diese Veränderungen in die Gesellschaft weiterträgt, spricht Putnam (2000) von bridging. Ausschlaggebend für den Erfolg sind letztlich der auf die Konfliktgruppen abzustimmende institutionelle Rahmen des Kontaktes (Salomon und Crains, 2010) sowie ein die Konfliktparteien verbindendes, meist konfliktneutrales Element, auch als Katalysator bezeichnet.

Dieser Beitrag will klären, wie sich die machtvoll etablierten Muster der Konfliktwahrnehmung durch Friedensbildung, die auf dem verbindenden Katalysator Umwelt aufbauen, verändern lassen und zu einer Transformation der Perspektiven auf den Konflikt beitragen. Dafür werden Sichtweisen von Studierenden nachgezeichnet, die sich durch ihre Studienzeit am Arava Institute for Environmental Studies (AIES) am Rande der Negev Wüste in Israel und durch die Teilnahme am anschließenden Alumninetzwerk in einem Prozess des bonding befinden. Die Operationalisierung des Perspektivenwechsels der Studierenden des AIES erfolgt über die Analyse konstruierter Raumbilder, die Aufschluss über die Wirkung und Strahlkraft von Friedensbildungsmaßnahmen geben.

Das gewählte Vorgehen nimmt sich verschiedener Lücken im interdisziplinären Feld der Konflikttransformation an. So ist das Potential der Umwelt als Instrument und Katalysator in der Konflikttransformation unbestritten, es scheint jedoch bei weitem noch nicht ausgeschöpft zu sein, zudem mangelt es an Fallstudien auf Graswurzelebene (Dabelko und Rogers, 2014; Kyrou, 2007; Conca und Wallace, 2012). In Bezug auf die Wahrnehmung von Konflikten stellen exemplarisch Bekermann (2011), Smith (2010), Salomon (2009) und Vukosavljevic (2007) die Notwendigkeit der Berücksichtigung von Identitäten, des historischen Erbes (engl. heritage) und territorialer Fragen, Orten mit besonderer Bedeutung, sowie von Macht-Ungleichgewichten heraus. An diese Forschungsdefizite wird durch die Einbeziehung neuer Erkenntnisse der geographischen Konfliktforschung (für einen Überblick vgl. z.B. Korf und Schetter, 2015) und allgemein der Bedeutung von konstruierten Raumbildern als Operationalisierung von Wahrnehmung angeknüpft (Rothfuß, 2011; Gregory, 2004; Korf und Engler, 2007). Darüber hinaus hat der Beitrag zum Ziel, entscheidende Erfolgsfaktoren des Fallbeispiels im Hinblick auf den institutionellen Rahmen sichtbar zu machen.

Die zentralen Ergebnisse dieser Studie sind zum einen, dass neben einem gemeinsamen Interesse an Umweltthemen bzw. -problemen, also dem Katalysator, vor allem das isolierte Zusammenleben in der Wüste während der Studienzeit, die gezielte Durchführung von Mediationsgesprächen sowie die Pflege der geschaffenen gemeinsamen Identität durch ein sehr aktives Alumninetzwerk entscheidend zum Erfolg von Friedensbildungsmaßnahmen beitragen. Insbesondere zeigt sich, wie weit sich tatsächlich Veränderungen in Bezug auf die Wahrnehmung der anderen Konfliktparteien sowie Ansätze des Entschuldigens erkennen lassen, und wie offen auch über Machtungleichgewichte diskutiert wird. Zum anderen wird jedoch auch deutlich, wie persistent die Sichtweisen auf „die“ Geschichte und ihr Erbe im Vergleich dazu doch bleiben; der erste Schritt des bonding ist, wie durch das Eingangszitat bereits illustriert, also ungleich größer als der zweite Schritt des bridging hin zu eigener Initiative und Wirkung als Agent des Wandels.

\section{Natürliche Umwelt als Katalysator in Friedensprozessen}

Die wissenschaftliche Literatur, die sich mit den Zusammenhängen zwischen natürlicher Umwelt und Konfliktsituationen bzw. der Schaffung von Frieden auseinandersetzt, fokussiert vor allem auf divergierende Vorstellungen von Flächennutzung (auch Verfügungsrechte und Tragödie der Allmende), Aspekte der Umweltdegradierung und deren (externalisierten) Kosten sowie Konflikte im Rahmen der Frage nach Umweltsicherheit (Floyd, 2013; Hurley et al., 2002; Ruschkowski und Mayer, 2011). In der Mehrzahl der Beiträge wird dabei die Umwelt als Gegenstand und/oder Ursache von Konflikten im Spannungsfeld von „Schützen vs. Nützen“ angesehen. Verhältnismäßig wenige wissenschaftliche Arbeiten untersuchen den Ansatz, gemeinsame natürliche Ressourcen und naturnahe Räume als Instrument der Konfliktlösung bzw. Deeskalation einzusetzen. Dieser Blickwinkel wurde von Studien des Environmental Peacemaking (umweltbasierte Konflikttransformation) angeregt, in deren Mittelpunkt letztlich die Frage steht, ob die Zusammenarbeit an spezifischen Umweltproblemen Frieden fördern und regionale Stabilität herstellen kann (Dabelko, 2008; Ali, 2007; Conca und Wallace, 2012). Insbesondere Conca und Dabelko (2002:9) heben dabei explizit das Potential der natürlichen Umwelt als Katalysator in Friedens- und Versöhnungsprozessen hervor:
While conflict and violence still dominate the en- vironmental security discourse, new research focu- sing on environmental peacemaking has challen- ged the assumed link to conflict [...] Environmental cooperation can be an effective general catalyst for reducing tensions, broadening cooperation, foste- ring demilitarization, and promoting peace.

Dieser Sichtweise zufolge kann Kooperationen in Zusammenhang mit der Lösung von Umweltkonflikten durchaus eine Rolle als Instrument zur Krisenprävention zugesprochen werden, insbesondere dann, wenn positive Effekte der Lösung des Umweltkonflikts auf andere Konfliktbereiche aus- 
Tabelle 1. Umwelt und Konflikttransformation: Konzeptionelle Ansätze.

\begin{tabular}{lll}
\hline Ansatz & Inhalte & Rolle der Umwelt \\
\hline $\begin{array}{l}\text { Politische Ökologie, Hazard- } \\
\text { Forschung, Livelihood-Ansätze }\end{array}$ & $\begin{array}{l}\text { Lösungsstrategien für Umweltkonflik- } \\
\text { te auf Basis des Gegensatzes, „Schützen } \\
\text { vs. Nützen“ }\end{array}$ & Ursache/Gegenstand von Konflikten \\
\hline Environmental Peacemaking & $\begin{array}{l}\text { Kooperation bei Umweltkonflikten } \\
\text { wirkt sich positiv auf andere Konflikt- } \\
\text { themen aus }\end{array}$ & $\begin{array}{l}\text { Teil des Konfliktes mit Potential für po- } \\
\text { sitive Spill-over-Effekte }\end{array}$ \\
\hline Peace Ecology & $\begin{array}{l}\text { Gezieltes Instrument zur Konflikttrans- } \\
\text { formation ohne spezifische Umwelt- } \\
\text { konflikte }\end{array}$ & \\
\hline
\end{tabular}

Quelle: eigene Zusammenstellung

strahlen, sich also positive Multiplikatorwirkungen entfalten. Erkenntnisse dazu liegen primär zu Fragen des grenzüberschreitenden Wassermanagements vor (Conca und Dabelko, 2002; Houdret, 2010); empirische Ergebnisse auf lokaler bzw. Graswurzel-Ebene gibt es dagegen kaum.

Ein nochmals weiterführendes Konzept in der Diskussion um den Friedens-Umwelt-Nexus ist der noch junge, vor allem in Arbeiten von Kyrou (2007) entwickelte Ansatz der Peace Ecology. Diesem Ansatz zufolge lassen sich die natürliche Umwelt und entsprechend umweltbasierte Friedensbildungsmaßnahmen auch unabhängig von ökologischen Fragen als Instrument der Konflikttransformation einsetzen. Zentrale Annahme ist dabei, dass Konfliktgruppen angesichts eines gemeinsamen Interesses an einer ökologischen Problemstellung eine Vertrauensbasis für die Zusammenarbeit bei weiteren Konfliktthemen aufbauen. In anderen Worten wird in dieser Konzeption die natürliche Umwelt als möglicher Katalysator für Friedensprozesse gesehen - auch ohne, dass ein spezifischer Umweltkonflikt, also konfligierende Interessen und Vorstellungen zur Nutzung eines bestimmten Gebiets und seiner naturräumlichen Potentiale, vorhanden sein muss. Umweltprobleme stellen also nicht mehr den Ausgangspunkt oder einen gewichtigen Teil von Konflikten dar, sondern eine wesentliche Möglichkeit, sie zu überwinden (vgl. auch Tab. 1).

Wie bereits mehrfach belegt, bergen Institutionen der Umweltbildung und ökologisch orientierte Ausbildungsstätten, die nicht direkt mit einem bestimmten Umweltproblem in Zusammenhang stehen (z.B. das Umweltprogramm der Vereinten Nationen, Ökomuseen), durchaus Potentiale zur Ausund Versöhnung beteiligter Konfliktgruppen (Davis, 1999; Kyrou, 2007). Die natürliche Umwelt ist bei solchen Initiativen zum einen ein physisches und/oder soziales Objekt, zum anderen werden ihr als ein kulturell konstruierter Raum bestimmte Werte zugeschrieben (Butz und Eyles, 1997; Ingold, 2000). Diesen Aspekten spürt der vorliegende Beitrag weiter nach; anders formuliert richtet die Studie besonderes Augenmerk auf die Umwelt als Katalysator in Prozessen der
Konflikttransformation. Auf der Grundlage des vorgestellten Peace-Ecology-Konzepts wird die operative Arbeit des Arava Institute for Environmental Studies in Israel auf das Potential hin untersucht, Umweltinhalte in einem Friedensbildungsprozess einzusetzen. Neben den Forschungen auf persönlicher, individueller Ebene werden institutionelle Faktoren der ausgewählten Fallstudie aufgedeckt, die eine Veränderung der individuellen Wahrnehmung beeinflussen können.

\section{Zur Operationalisierung von Konfliktwahrnehmung}

Die Änderung von Wahrnehmungen und mögliche Perspektivverschiebungen von Akteuren, die an einer Friedensbildungsmaßnahme beteiligt sind, lassen sich grundsätzlich auf verschiedenen Wegen beschreiben. Die vorliegende Studie legt ihr besonderes Augenmerk auf die Wirkmacht raumbezogener Repräsentationen, die in der geographischen Konfliktforschung verhandelt werden. Hier nehmen die Beurteilung subjektiver Wahrnehmungen und Perspektivenwechsel im Zuge der Friedenssicherung sowie die Konstruktion bzw. Rekonstruktion von sozialen und physischen Räumen und deren Relation zu vorhandenen oder sich verändernden Machtgefügen eine gewichtige Rolle ein (Watts, 2000; Bohle, 2004; Korf und Engeler, 2007; Reuber und Strüver, 2009; Rothfuß, 2011). Korf und Raeymaekers (2012) zeigen in diesem Zusammenhang, dass selbst in gewaltoffenen Auseinandersetzungen Räume der sozialen Ordnung entstehen können, die einen Fortbestand gesellschaftlichen Lebens durchaus ermöglichen. Ein wesentliches Merkmal dieser (re-)produzierten (Raum-)Bilder ist ihr performativer Charakter (Van Gielle Ruppe et al., 2012). Inwiefern etablierte Raumkonstruktionen im Zuge der Zeit veränderbar sind, wie entstandene Machtgefüge sich neu verteilen lassen und welche Relevanz Machtungleichgewichte in diesem Zusammenhang haben, ist folglich Gegenstand zahlreicher wissenschaftlicher Beiträge (z.B. Castree, 2007; Springer, 2011). 
In Arbeiten der geographischen Konfliktforschung fällt drei Kategorien von konstruierten Raumbildern eine besondere Bedeutung $\mathrm{zu}$, in denen auch etablierte Konzepte der Friedensbildung wie differenzierte Gewaltformen (Bastien et al., 2003; Galtung, 1996; Vickers und Matthews, 2002) und Ansätze zur Aus- und Versöhnung (Lederach, 1997; Buckley-Zistel und Moltmann, 2006) Berücksichtigung finden. Erstens nehmen „Gruppen“ (z.B. Ethnien, soziale Schichten) eine wichtige Rolle für die Konstruktion und Instrumentalisierung von Differenzen und Deutungshoheiten ein (Korf und Engeler, 2007). Zentral sind dabei Zuschreibungen für die jeweils ,,andere“ Konfliktpartei. Veränderungen der Perspektive würden damit ein Auflösen der Polarisation von „Wir“ und „Die“, eine vielschichtige Wahrnehmung der „Anderen“ und der Wunsch nach friedlicher Koexistenz der Gruppen bedeuten.

Zweitens repräsentiert „Territorium“ die Konstruktion und die Bedeutung von physischem und politischem Raum (Cox, 2008), wie z.B. Doevenspeck (2011) an der Grenze zwischen Ruanda und der Demokratischen Republik Kongo aufzeigt. Im Hinblick auf die Konfliktwahrnehmung fallen hier die Darstellung und Bedeutung von Grenzen, die Formulierung von Ansprüchen auf Räume und Ressourcen sowie die Zugänge zu Räumen ins Gewicht. So diskutiert etwa Korf (2012), dass die Knappheit an natürlichen Ressourcen nicht zwingenderweise Konflikte anheizt, sondern auch Anreize zu Kooperation und Annäherung bringen kann.

Drittens steht das historische/kulturelle „Erbe“ (Heritage), das unterschiedlich inszeniert bzw. interpretiert werden kann, stark in Verbindung mit Machtdiskursen (Rodrian, 2011; Van Gielle Ruppe et al., 2012). Für die Frage nach einem Perspektivenwechsel sind die Anerkennung von Verletzungen der eigenen und der anderen Seite in der Vergangenheit, die Instrumentalisierung der Geschichte für Ansprüche und Konflikthandlungen oder die gemeinsame Version der Vergangenheit von Bedeutung.

Um die individuelle Sichtweise der Teilnehmerinnen und Teilnehmer an einer Friedensbildungsmaßnahme auf die Konfliktursache aufschlüsseln zu können, liefern diese Kategorien entscheidende Informationen zur Transformation der zu lösenden Konfliktgegenstände. Folglich kann von einer erfolgreichen Veränderung der Perspektive gesprochen werden, wenn sich die Wahrnehmung in diesen Feldern verändert.

\section{Untersuchungskontext und methodisches Vorgehen}

Ausgehend von den etablierten Instrumenten des Konfliktmonitoring nach Wehr (1979), Wilmot und Hocker (2001) sowie Kyrou (2007) wurde für die vorliegende Untersuchung ein Konfliktmonitoring-Leitfaden konstruiert, der die oben genannten Konzepte zusammenführt, um den Einfluss und die Wirkung von Friedensbildungsmaßnahmen in einem

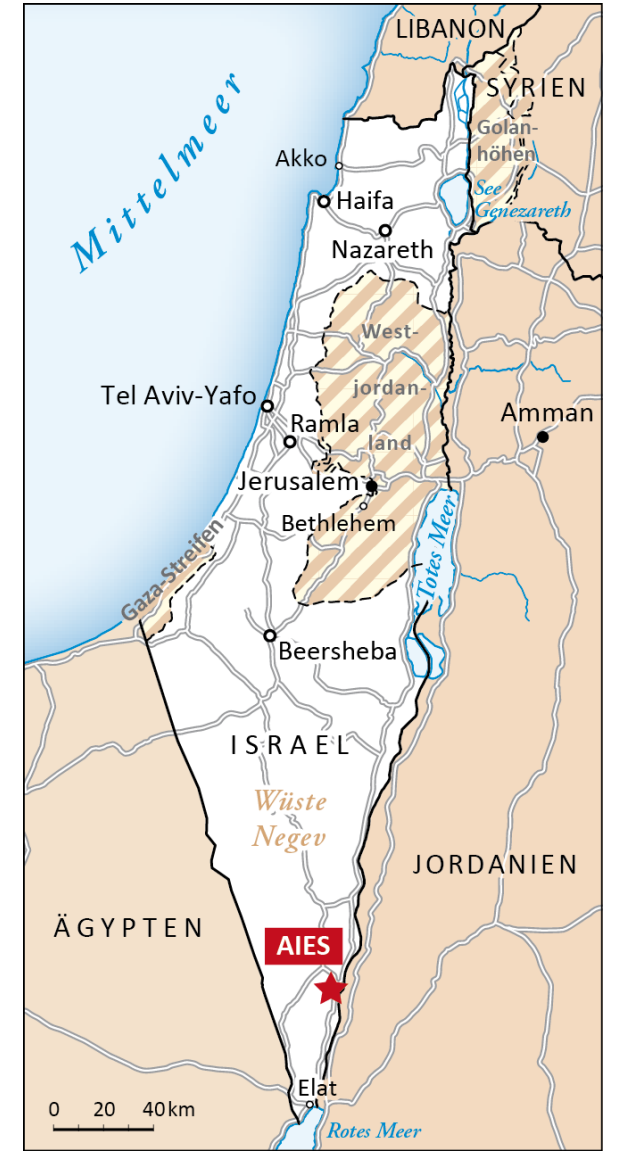

Abb. 1. Standort des Arava Institute for Environmental Studies (AIES), Israel (Kartographie: Alexandra Kaiser 2015).

Konflikttransformationsprozess zu ermitteln (siehe ausführlicher Rodrian et al., 2015). Dieser Leitfaden diente als Grundlage für die empirischen Arbeiten während zwei Forschungsaufenthalten in Israel, Jordanien und den palästinensischen Autonomiegebieten im Mai/Juni 2014 und im Mai 2015.

Im Fokus der Untersuchungen standen die Studierenden und Alumni des Arava Institute for Environmental Studies (AIES) in der Negev Wüste auf dem Kibbuz Ketura im Grenzgebiet zwischen Israel und Jordanien (Abb. 1). Das AIES verfolgt die Ziele, zukünftige Führungspersonen aus dem Nahen Osten in Umweltfragen auszubilden und dabei gleichzeitig einen Dialog zwischen den Konfliktparteien zu initiieren. Seit der Gründung im Jahr 1996 haben 857 Personen ein Studium am AIES absolviert; aktuell sind 32 Studierende eingeschrieben (Stand Oktober 2015). Es versteht sich also nicht vorrangig als Friedensinitiative, sondern als Institution der Umweltbildung, welches den Studierenden ermöglicht, sich über ein neutrales Thema zu begegnen.

Das empirische Material basiert auf 16 problemzentrierten Expertengesprächen mit Entscheidungsträgern und dem Lehrpersonal des Arava-Instituts, 25 narrativen Interviews und zahlreichen teilnehmenden Beobachtungen und infor- 
mellen Gesprächen mit Studierenden und Alumni von Arava, sowie Auswertungen von Chats und Diskussionen in Facebookgruppen und weiteren Dokumenten (z.B. monatlicher Newsletter des Arava-Instituts). Die Beobachtungen erfolgten bei laufenden Mediationsrunden, im Kontext von Alumnikonferenzen und auf Exkursionen mit den Studierenden. Besonders im Blick standen Wahrnehmungszustände und -änderungen der Beteiligten und die Evaluation der Friedensbildungsmaßnahme hinsichtlich ihrer Wirkung auf die Konfliktparteien. Dabei stellte sich die Befragung der Alumni als besonders wertvoll heraus, um sowohl einen Ist-Zustand nach dem Durchlaufen der Maßnahme festzustellen als auch die Retrospektive der Alumni auf ihre Zeit am Arava-Institut zu ergründen.

Die Auswertung des transkribierten bzw. in Gedankenprotokollen übertragenen empirischen Materials zeigt in der Kodierung drei Hauptkategorien, die den empirischen Teil gliedern: Die Bedeutung des Umfeldes des AIES einschließlich des Katalysators Umwelt, die Veränderung der Wahrnehmung auf den Konflikt - hier spiegeln sich die Kategorien der Operationalisierung wider - sowie Rückkehr der Studierenden in ihr alltägliches Umfeld nach dem Aufenthalt am AIES.

\section{Empirische Befunde}

Die Konflikttransformation des AIES arbeitet mit einem Wechselspiel von Orten und Räumen des Friedens und des Konflikts. Aus räumlicher Perspektive betrachtet rücken Orte des Austausches, Räume des (isolierten) Zusammenlebens inmitten der Natur auf dem Campus in der Wüste, aber auch gezielt konstruierte Räume der verbalen Konfrontation und gemeinsame Exkursionen zu sensiblen Orten kriegerischer Auseinandersetzungen ebenso wie Räume des Zusammenhalts auf jährlich stattfindenden Alumnikonferenzen in den Fokus der Betrachtung. Unter Einbeziehung der Umwelt als Katalysator, der ,keine Grenzen kennt“ und den thematischen sowie physischen Rahmen der Maßnahme darstellt, schnüren diese Plattformen das Transformationspaket des Arava-Instituts. Durch die damit eingeleiteten Prozesse verändern sich Einstellungen zu den anderen Konfliktgruppen und die Wahrnehmung auf den Konflikt. Eine Transformation der Sichtweise auf das eigene Erbe gelingt jedoch kaum; auch sind der Übertragung in reale Handlungen bzw. der Wirkung als Multiplikator enge Grenzen gesetzt.

\section{Umwelt als Katalysator: Interaktion an Orten des Konflikts und Räumen des Friedens}

Die Studierenden leben für mindestens ein Semester auf dem Campus des AIES. Dieser von der Außenwelt abgeschiedene Ort und die damit verbundene räumliche und soziale Isolation tragen entscheidend zur Konflikttransformation bei. So berichten die Teilnehmerinnen und Teilnehmer am Pro- gramm des AIES zum einen von einem intimen Prozess der Selbstfindung und Selbstreflexion, der durch die äußeren Begebenheiten eines Lebens inmitten der Natur gefördert wird. Zum anderen stellt die Abgeschiedenheit - oder wie Interviewpartner es formulieren ,die freiwillige Gefangenschaft" - in der Wüste die Basis für Austausch und auch Auseinandersetzung dar. Das gemeinsame Leben in der Isolation bildet somit die Grundlage für Dialog und Austausch: ,[T]here is always someone around and there is no way out, but you easily meet like-minded fellows ... [F]riendship is just a consequence of that environment" (Palestinänsischer Alumnus, Interview, Mai 2014). Dabei bringt die Abgeschiedenheit jedoch nicht nur Kontakte und Freundschaften mit sich. Das Reden, Streiten und Diskutieren über den gemeinsamen Konflikt ist omnipräsent, unbequeme Themen sind dabei stets Inhalt des Diskurses:

The Arava experience shifts people's thinking, and this translates into action. Dialogue at the Arava was neither feel-good, nor easy, and uncomfortable yet powerful dialogue is one of the many keys to moving the situation forward (Alumna aus Israel, Director's Blog AIES, Juli 2014).

Der Zustand der Isolation in der Wüste wird von einer weiteren Maßnahme flankiert, die eine Auseinandersetzung mit dem Gegenüber fördern soll. So wird das Zusammenleben der Studierenden bewusst in Wohngemeinschaften organisiert, die aus unterschiedlichen Konfliktgruppen und -kulturen stammen. Dies fördert den Prozess des bonding und ermöglicht das Entstehen sozialer Bindungen und Freundschaften über Konfliktgrenzen hinaus:

I shared my room with a guy from Jordan and an Israeli. Even though there is very little privacy, I must say that the concept of shared rooms is essential to get to know the opposite culture. I learned so much about Jews, and it was nice to also speak about my culture in such an intimate setting" (Palestinänsischer Alumnus, Interview Mai 2014).

Die Kombination aus räumlicher Isolation in der Natur und den bewusst geschaffenen Orten der alltäglichen Interaktion fördert den Aufbau von sozialem Kapital. „I didn’t know Jewish people or have Jewish friends. Now, because of Arava, two of my best friends are Jewish" (Palestinänsischer Alumnus, Webseite AIES, März 2014).

Das räumliche Setting aus „Gefangenschaft in der Wüste“ und Interaktion bringt auch Konfrontation mit sich. Zur Kanalisierung der Konflikte müssen die Studierenden an dem sogenannten Peacebuilding and Environmental Leadership Seminar (PELS) teilnehmen, das Gastvorträge, geführte Diskussionen, Workshops, Mediationskurse und Exkursionen beinhaltet. Auch in diesen Kursen hilft der Katalysator Natur, um über konfliktrelevante Umweltthemen ein gemeinsames Interesse der Studierenden anzusprechen und davon ausgehend auf allgemeine Konfliktthemen überzuleiten. Oftmals 
waren sich die Studierenden im Vorfeld nicht über Existenz und Umfang der Mediation im Curriculum bewusst und wurden entsprechend überrascht. Nach den Berichten der Studierenden stellt dieses Seminar eine große Herausforderung für sie dar, da sie bewusst aus ihrer „Komfortzone“ getrieben werden. Die geschützten Räume des PELS ermöglichen es, in Ruhe über den Konflikt zu sprechen und die anderen Seiten zu hören bzw. hören zu müssen:

Many students contended that they were here to study about the environment and they don't want to be part of a discussion about the conflict. Some were sure that this is a useless exercise and that there is no one ready to hear their words on the other side. But they all agree, by the end of the semester, that PELS was a significant component in the program; it is the glue that keeps the different parts of our experience together (Alumnus aus Jordanien, Director's Blog des AIES, Juli 2014).

Die Versöhnung und Aufarbeitung sozialer Beziehungen ist ein elementarer Bestandteil der Friedensbildung. PELS vermittelt Kommunikationstechniken wie „compassionate listening“, die für die Aufarbeitung von Konfliktgegenständen dienlich sind. Aus teilnehmenden Beobachtungen und Interviews lässt sich schließen, dass die Studierenden des AIES sich ein Instrumentarium an Fertigkeiten und Regeln angeeignet haben, die eine Kommunikation auf Augenhöhe erleichtern.

If everyone had PELS, no one would be chanting "Death to Arabs", or cheering when rockets and bombs caused injuries and deaths, because PELS allowed us to humanize the "other". Despite our disagreements, we were all able to see each other as sapient beings, the same as ourselves (Alumnus aus Israel, Director's Blog AIES, Juli 2014).

Besondere Interventionen sind Exkursionen zu historisch bedeutenden und konfliktrelevanten Orten. Durch das bewusste Aufsuchen von Orten des Konfliktes im Wechsel zum geschützten Ort des Friedens, gibt AIES den Studierenden die Möglichkeit, Orte des Konflikts gemeinsam zu erleben und zu reflektieren.

Die Bedeutung der sicheren und geschützten Räume und Orte wird immer wieder in Gesprächen aufgegriffen. Entscheidend ist dieses Konzept nicht nur während der Studienzeit am AIES, sondern auch danach. Da die Teilnehmerinnen und Teilnehmer oftmals sozialem Druck ausgesetzt sind und sich in ihrem gesellschaftlichen Alltag hilflos, ohnmächtig und verschiedenen Formen der (nicht-physischen) Gewalt ausgesetzt fühlen, spielt das Alumninetzwerk eine entscheidende Rolle zur Bewahrung der Erfolge der Konflikttransformation. Regelmäßige Alumnitreffen und eine Facebookgruppe begleiten den Alltag der Studierenden meist über Jahre hinweg. Somit besteht die Möglichkeit, negative Reaktionen des Umfelds oder exogene Schocks, wie kriegerische
Auseinandersetzungen der beteiligten Länder, zu moderieren. „The Alumni Network is most probably the most powerful tool of Arava [AIES]. It assists us in the further process of peace" (Programmdirektorin Cathie Granit, Interview, Mai 2014). Die Regelmäßigkeit von Veranstaltungen und der tägliche digitale Austausch über die Facebookseite des Alumninetzwerks stellt eine temporäre Komponente dar, die entscheidend zur Transformation des Konfliktes auf Graswurzelebene beiträgt, indem Erfolge konserviert werden und der Dialog aufrecht erhalten bleibt.

Die interviewten Studierenden entschließen sich in der Regel zum AIES zu kommen, um sich Fachwissen im Bereich der Umweltwissenschaften anzueignen. Ziel fast aller Interviewpartner war ihre wissenschaftliche Ausbildung weiter zu gestalten und nicht an einer Friedensbildungsmaßnahme teilzunehmen. Die Umweltforschung wirkt dabei in direkter und indirekter Weise als Katalysator: In direkter Weise im Sinne des Multiplikator-Effekts, etwa mit Ansätzen im Bereich grenzüberschreitendes Wassermanagement, im Zuge dessen eine Region im Interesse aller Parteien und ökologisch nachhaltig mit Wasser versorgt wird. Die Konzipierung unterschiedlichster Modelle zur Lösung der Wasserknappheit regt die Studierenden an, sich mit den Interessen der Konfliktparteien auseinanderzusetzen. Die wissenschaftliche Herangehensweise an diese Problematik führt dazu, dass die Studierenden einen ungefärbten und lösungsorientierten Blickwinkel einnehmen können.

Indirekt liefert die wissenschaftliche Auseinandersetzung mit regionalen Umweltproblemen einen neutralen Gegenstand für Austausch und Zusammenarbeit. Der natürlichen Umwelt wird dabei die entscheidende Rolle als Medium und Katalysator in der Konflikttransformation zugesprochen:

As we all know, we can't deny the fact and also the beauty that nature really knows no boarders. Don't we all see and experience that the environment is struggling so badly from water scarcity in this region? Shouldn't we all become ambassadors for the environment in the Middle East and take this as an opportunity to overcome our violent relationships? (Alumna aus Israel, Interview Mai 2015).

Der Schutz der Umwelt und die wissenschaftliche Ausbildung in der Umweltforschung stellt das entscheidende gemeinsame Interesse aller Studierenden dar. Gleichzeitig wird der Katalysator mit dem Slogan von AIES ,nature knows no boarders" als Vorbild für weitere Prozesse gesehen, da nicht nur Umweltprobleme frei von politischen Grenzen sein können, sondern auch andere Themen über Grenzen hinaus wirken. Weiterhin beschreiben Interviewpartner neben dem zusammenführenden Effekt des „Gefangenseins“ in der Natur explizit die Hügel in der Umgebung des Campus als wichtigen Teil des AIES-Umfeldes, die als Rückzugsraum dabei helfen, Maßnahmen wie PELS zu verarbeiten bzw. sprichwörtlich „,von oben“ zu betrachten: ,Desert life forces you to reconsider your actions.[...] Without my regular walks out- 
side the Kibbuz into these beautiful majestic mountains I would have drowned at Arava. It was important for me to have time for myself to reflect on the things that were going on. These quiet walks into the sandy rocks helped me to actually re-build my personality.“ (Alumnus aus Jordanien, Interview, Mai 2015). Die Natur als „Ruheort“ leistet folglich einen weiteren indirekten Beitrag zur Veränderung der Wahrnehmung, da sie die persönliche Reflexion der Studierenden fördert.

\section{7 „Das ist mein Land und mein Erbe“ - Zur Veränderung von Wahrnehmungen}

Eine Veränderung der Wahrnehmung auf Konflikte kann durch die Analyse von Interpretationen raumbezogener Repräsentationen sichtbar gemacht werden. Dabei wird ein Bruch deutlich, der zwischen der neu geformten Gruppenidentität sowie dem Wunsch nach Frieden auf der einen Seite und der Persistenz des Erbes der Vergangenheit sowie fehlender Anerkennung des Leides der anderen Konfliktpartei auf der anderen Seite verläuft.

Durch die Konfrontation mit anderen Konfliktakteuren in gezielten Mediationsmaßnahmen wird die Rekonstruktion eigener Denkmuster und Handlungsweisen angeregt. Wie folgende Ausführungen einer Studierenden aus Israel veranschaulichen, können die Maßnahmen eine Veränderung der eigenen Wahrnehmung auf den Konflikt und seine Akteure zur Folge haben:

I did not leave PELS sessions feeling calm and contemplative. Rather, I walked out slowly because I was processing my rising bitterness for not having previously questioned enough ... The demands in my mind would persist; why didn't I ask my parents more about what it meant to be born on a kibbutz in the north between Arab villages? Why, when I was younger, was I silent and self-conscious during Jewish history classes when a friend would enquire, 'but what about the $\mathrm{Pa}-$ lestinian refugees?' Why, through the years at the youth movement that I was active in, did I not engage more deeply with the implications of leftwing Zionism? Throughout my life, I had keenly selected other difficult issues to pick apart - why not these ones? Perhaps it is because this process of learning, unlearning and coming to peace with a radically new framework of understanding invokes guilt and confusion and demands a jarring reframing of identity. Either way, integrating these issues with a group of people engrossed in the situation is an experience that countless young Jews embedded in Zionist communities both in Israel and abroad do not often go through, and it is an experience for which I am extremely grateful (Director's Blog des AIES, Juli 2014).
Die Alumna hinterfragt ihre gesellschaftliche Einbettung, sowohl auf familiärer als auch auf institutioneller Ebene. Sie sieht eine Diskrepanz ihrer eigenen Moralvorstellung mit ihrer kulturellen Herkunft und kritisiert ihre linear erfahrenen Erziehungsinhalte. Dieses Ungleichgewicht ist das Ergebnis einseitiger und intransparenter Vermittlung von Identität, was ein klares Indiz für epistemologische und strukturelle Gewalt im Bildungs- und Erziehungssystem ihres Herkunftslandes darstellt. Zudem veranschaulicht das Zitat, dass innerhalb des Transformationsprozesses eine Neukonstruktion der persönlichen Wahrnehmung erfolgen kann - eine notwendige Grundlage zur Aus- und Versöhnung sowie für einen Perspektivenwechsel der Konfliktakteure.

Durch die Friedensbildungsmaßnahme gelingt es, den beteiligten Konfliktakteuren eine gemeinsame Identität zu vermitteln und den Prozess des bonding zu initiieren. Dazu gehören ein gegenseitiges Verständnis, eine neue Definition von Gruppen unter den Studierenden und der Aufbau von Vertrauen. Elementar sind das Entstehen einer gemeinsamen Gruppenzugehörigkeit und das Auflösen von Machtstrukturen durch die Gleichbehandlung aller Teilnehmerinnen und Teilnehmer. Dieser soziale Schutz stärkt die Gruppendynamik und fördert die Gruppenwahrnehmung, wie es ein Alumnus aus Palästina ausdrückt: "The people there are special. Everyone was equal. Everyone was the same" (Palestinänsischer Alumnus, Interview, Mai 2014).

Die bei vielen Interviewpartnern ursprünglich vorhandene sehr homogene Betrachtung der anderen Konfliktparteien ersetzt sich durch eine differenzierte und weniger verallgemeinernde Sichtweise. „When you tear down these mental fences, you start realizing that you deal with people. There is no we and there is no they anymore" (Israelischer Alumnus, Interview, Mai 2015). Entsprechend wird auch unter den externen Einflüssen des Krieges 2014 auf den Blogs immer wieder die gemeinsame Gruppenidentität beschworen:

In these politically complicated times, we need to remember that we are all humans and not to let hatred control us! We need to remember the similarities that bring us together and not focus on the differences that push us apart (Alumna aus Israel, Director's Blog des AIES, Juli 2014).

In Bezug auf territoriale Raumbilder, die eine Schlüsselfrage des Konflikts darstellen, sind in vielen Interviewpassagen Veränderungen der Wahrnehmung zu erfahren. Dazu gehört die Einstellung, etwas verändern zu müssen und den Weg einer Zwei-Staaten-Lösung zu suchen.

We can not move on like this. There is no way Arabs will win and there is no way Jews will win this conflict. What kind of life are people living in the Middle East. The quality of life is actually low here, because of the territorial and ethnic hassle all the time - some might disagree on this, because they are used to it, but actually it is the case. Arabs 
and Jews have formed this region, so why not give both nations a piece of the cake (Palestinänsischer Alumnus, Interview, Mai 2014).

Neben Aussagen zu der Zwei-Staaten-Lösung fallen Interviewpassagen auf, die innenpolitisch auf eine Veränderung der jeweiligen Gesellschaften abzielen und die territoriale Fragen aufzeigen. Da es vielen arabischen Studierenden aus Israel beispielsweise untersagt ist, sich frei in Israel zu bewegen, müssen sie auch die vorlesungsfreie Zeit ausschließlich auf dem Campus des Instituts verbringen. Dadurch, dass jüdische Studierende das miterleben, wurde ihnen die andere Perspektive klar, was ihre Wahrnehmung bezüglich eingeschränkter Handlungs- und Bewegungsspielräume und vorhandener Machtungleichgewichte in der israelischen Gesellschaft verändert hat. Ein israelischer Alumnus beschreibt:

We have to do our homework first and stop our racist patterns in Israel itself. Arab citizens of Israel are living under very bad conditions in this country. Some can not move around freely and their political rights are being withheld, that is what you call Apartheid. Israel has to improve the living conditions of Israeli Arabs and close the massive gap between Jewish and Arab Israelis first and then we can talk about other solutions (Interview, Mai 2015).

Folglich lassen sich neben Veränderungen in der Wahrnehmung von Gruppen in territorialen Fragen Perspektivwechsel und Reflexionsprozesse beschreiben. Im Gegensatz dazu verweisen Studierende unabhängig ihrer Herkunft immer wieder auf die Geschichte und ihr Erbe:

I am not saying that I am right wing, but I believe that Zionism is the only answer to what happened to the Jews in the Holocaust. There is no other nation that deserves peace more desperately then the Jewish one. And that is what Arabs need to understand (Israelische Alumna, Interview, Mai 2014).

Dieser Interviewausschnitt zeigt, wo die Grenzen des Transformationsprozesses der individuellen Wahrnehmung verlaufen können. Eine Einschränkung der eigenen Ansprüche auf Basis des eigenen Erbes bzw. eine gleichgewichtige Anerkennung des ,,anderen“ Erbes stellt eine große Hürde der Friedensbildungsmaßnahme dar. Hier wird die in der Diskussion um die Wahrnehmung als Gruppe aufgelöste Konstruktion des ,wir“ und ,die anderen“ wieder deutlich instrumentalisiert.

Auch für den Perspektivenwechsel auf das Leid der anderen Konfliktseite nimmt der Bezug zur Geschichte und die Konstruktion von ,wir“ und ,die anderen“ eine starke Position ein, wie eine ehemalige Studentin aus Palästina in der Facebookgruppe postet:

To all out there: stop equalizing the pain, the suffering, the killing and the responsibility. We are not equal sides, this is not a conflict, this is Israel occupying people and depriving us of our right to exist as equal human beings (Palästinensische Alumna, Alumni Facebookgruppe, Juli 2014).

Darin zeigt sich exemplarisch die Diskrepanz, das Leid der anderen Seite in der Vergangenheit anzuerkennen und neben das eigene erlittene Unrecht zu stellen. Dieser Schritt wäre für einen Transformationsprozess jedoch ein entscheidender Meilenstein, da die Legitimierung von zukünftigen Handlungen auf historischen Ansprüchen und den eigenen Verlusten basiert. Gemäß den empirischen Einsichten gelingt dies nur innerhalb der Gruppe und mit Blick auf den zukünftigen Wunsch nach Frieden, kaum jedoch im Hinblick auf das Erbe und Schritte hin zur gleichgewichtigen Anerkennung von Verletzungen und Leid. Die eigene „Wahrheit" wird hier als die überlegene, die wahre Geschichte angesehen. Damit besteht ein Gegensatz zu der in allen Interviews beobachteten Sehnsucht nach Frieden in der Zukunft und nach einer Neustrukturierung des Umfelds.

Exemplarisch für den Bruch zwischen der gemeinsamen Gruppe und dem Wunsch nach Frieden sowie territorialen Fragen auf der einen Seite und dem eigenen Erbe sowie dem Mangel an Perspektivwechsel für erlittene Verletzungen auf der anderen Seite steht eine heftige Auseinandersetzung eines israelischen und eines palästinensischen Alumnus auf der Facebookgruppe des AIES. Auf einer späteren Alumnikonferenz kommt es in einer geführten Mediationsrunde zur Aussprache, der folgendes Zitat entnommen ist:

I want to take this opportunity to truly apologize for the pain I caused you. You can do whatever you want with the words I am telling you now. I got to know you some time ago and I got to like you, nevertheless our cultural differences are standing in between us. I am telling you right now, that I respect your point of view and that I respect your culture. I disagree on most of your arguments, but still I believe we should just accept our different realities and move on in peace (Israelischer Alumnus, Teilnehmende Beobachtung Mediationsworkshop, Mai 2015).

Der Wunsch nach gegenseitigem Respekt, nach Einheit und Miteinander in der gemeinsamen Gruppe und nach einer anderen Zukunft sprechen mit. Hier zeigen sich die Möglichkeiten, aber auch die Grenzen der Friedensbildung. Der Dialog bleibt bestehen, ebenso das Gruppengefühl und der Wunsch nach Frieden; das gemeinsame Suchen nach einer Wahrheit und einer reflektierten Version der Geschichte und des Erbes erfolgt nicht. Die Option, zwei unterschiedliche Wahrheiten anzuerkennen, fand positiven Anklang und ist ein Zeichen einer veränderten Wahrnehmung, wenn auch mit Grenzen. 


\section{Grenzen des Brückenschlagens}

Bezugnehmend auf das zu Beginn des Beitrags angeführte Zitat stellt die Übertragung der geänderten Wahrnehmung in Handlungen und auf weitere Akteure die größte Herausforderung dar. Viele Studierende sehen sich mit gewohnten Strukturen und Hürden - sowohl in familiären Kreisen als auch auf politischer Ebene - konfrontiert, die nicht zu überwinden sind. Dabei berichten Alumni auch von persönlichen Krisen, wenn die eigene, veränderte Einstellung im sozialen Umfeld auf Widerspruch stößt. So besteht eine Diskrepanz zwischen der reflektierten Wahrnehmung der Alumni auf den Konflikt und seine Akteure und den Begebenheiten außerhalb des geschützten Raumes:

At the end of the semester, I felt equal, I felt empowered to change, I felt I was a leader; I was ready to start my future with bigger hopes. However, the harsh reality slapped me in the face. The skills I had gained in PELS have no place in my current reality. I can't practice them because there are gaps which need to be filled; there are basics that don't exist within our societies. At this time, we cannot sit at the same table to work out our differences if one party is still occupied and the other is the occupier (Palästinensische Alumna, Interview, Juni 2015).

Neben den alltäglichen Hürden im engen und erweiterten sozialen Umfeld nehmen exogene Schocks, wie die kriegerische Auseinandersetzung Operation Protective Edge im Sommer 2014, Einfluss auf die Dauerhaftigkeit und Strahlkraft der Friedensbildungsmaßnahme. Eine palästinensische Alumna äußerte sich während dieses 50 Tage andauernden Gaza-Krieges verzweifelt und beschwört im geschützten Facebookchat die gemeinsame Gruppenidentität und deren weitere Existenz:

When I see this sad news I remember when I looked at us in AIES with eyes full of tears when we played a football game students against staff. People from all nationalities playing in one team in one hand happily... When I saw u guys I wished I can video tape it and show the world a proof that we can live together in peace... sure we can if we really wanted... hope the dream peace will come true soon... (Palästinensische Alumna, AlumniFacebookseite, Juni 2015).

In dieser Einlassung zeigt sich die Herausforderung, die alleine durch die Aufrechterhaltung der veränderten Wahrnehmung erwächst, um nicht in vorherige Wahrnehmungsmuster zurückzufallen.

Der Bruch zwischen sozialem bonding der Studierenden und Multiplikatoreffekten durch bridging und direkte Handlungen wird auch in Interviews und auf den Alumniveranstaltungen deutlich. Eine Chance für Multiplikatoreffekte ist, im familiären und gesellschaftlichen Kontext als Vorbilder zu fungieren, um erlernte Sichtweisen in die Gesellschaft zu übertragen - auch wenn dieser Prozess nicht ohne Hürden ist:

After my studies at Arava I took one of my best Israeli friends to my parents' house. I was so nervous and we did not tell my family that she was Israeli. My parents immediately liked her and treated her like any other friend. When I told my mom that she is Israeli, she looked at me and told me: ,So what? I love her and you better make sure she will visit us again" (Palästinensische Alumna, Interview, Mai 2014).

Diese Geschichte des Erfolgs im Kleinen gibt Hoffnung für eine Übertragung der Konflikttransformation, zeigt zugleich aber auch die Schwierigkeiten und die Grenzen auf, mit denen sich die Alumni konfrontiert sehen. Die Mehrheit der Erzählungen ist geprägt durch Hemmnisse und Zweifel, ob und wie die Erkenntnisse von AIES weitergegeben werden können.

In Bezug auf konkrete Handlungsveränderungen sind in Interviews und durch Beobachtungen zahlreiche Neugründungen von Nichtregierungsorganisationen - wie Eco-Path, Youth Environmental Education Peace Initiative (YEEPI), Masar oder Arava Alumni Peace and Environmental Network (AAPEN) - zu verzeichnen, die von Alumni des AravaInstituts ins Leben gerufen wurden. Bei diesem Prozess wird auch wieder die Bedeutung des Katalysators Natur deutlich, da die Ausgründungen die Verknüpfung von Umwelt- und Friedensfragen übernehmen und weitertragen; nach Aussagen von Beteiligten sind vor allem Maßnahmen für Kinder und Jugendliche erfolgreich im Hinblick auf die Konflikttransformation. Hier eröffnen sich Handlungsspielräume für Alumni, als aktive Agenten der Friedensbildung zu wirken. Beide aufgezeigten Erfolgsgeschichten sind jedoch positive Ausnahmen im Prozess des bridging. Der überwiegende Teil der Interviews beschreibt die Hindernisse. Dadurch bleibt der Aufbau einer gemeinsamen Identität in einem Schutzraum, der durch digitale und physische Begegnungsräume aufrechterhalten wird und in seiner Strahlkraft nach außen stark limitiert ist. Innerhalb dieses Schutzraumes sind jedoch Veränderungen der Wahrnehmung der Beteiligten auf den Konflikt zu beobachten.

Zusammenfassend lässt sich feststellen, dass der Schritt in die „reale“ Welt, die von verschiedensten Formen der Gewalt geprägt ist, für einen Großteil der Studierenden am AIES ein Schritt in eine neue Welt ist. Die Perspektiven auf den gegebenen Konflikt und seine Akteure haben sich durch die Inhalte des Studiums geändert, die Realität indes scheint stehen geblieben zu sein. Um die Gruppenidentität der AravaAlumni zu schützen und zu stärken ist die Kontinuität des gegenseitigen Austausches über das Alumninetzwerk, auch in schwierigen Zeiten, ein unersetzliches Werkzeug in der langfristigen Transformation dieses Friedensprozesses. Hoffnung 
dazu gibt das bereits wiedergegebene Zitat des israelischen Alumnus, der neben seiner auch eine andere Wahrheit akzeptiert und den Fortbestand der Friedensabsicht beider in den Fokus rückt. Insgesamt betrachtet bleibt anzuerkennen, dass viele Aspekte eines Perspektivenwechsels nachweisbar und der Wunsch nach Frieden formuliert wird; auch die persönliche Transformation eigener Denkmuster und Handlungsweisen erscheint umsetzbar. Die größte Hürde, die es dabei zu überwinden gilt, ist das kulturelle Erbe und die darauf aufbauende Prägung, die tief in den Köpfen der Konfliktakteure verankert sind.

\section{Fazit}

Im Zentrum der Betrachtung stand die Frage, wie die Wahrnehmung von Track 3 Akteuren auf einen Konflikt verändert werden kann. Dabei ist zunächst der Weg zur Veränderung der Wahrnehmung zu analysieren. Umweltbasierte Friedensbildungsmaßnahmen können gerade in Räumen mit Ressourcenknappheit einen Beitrag zur Konflikttransformation leisten. Dadurch, dass Umwelt als existenzielle Ressource interpretiert wird, eröffnet sich ein Feld für Perspektivenwechsel und ein starkes gemeinsames Interesse. Die im konzeptionellen Teil vorgenommene Unterteilung der Natur als Katalysator und Multiplikator ergänzt sich in der Empirie durch die Natur als eine die Veränderung von Wahrnehmung fördernde räumliche Umgebung; konkret durch das „Gefangensein“ in der Natur und als Ruheort zur Reflexion der Mediationsrunden.

Parallel zur Bedeutung der Umgebung des Transformationsortes sind die von den Studierenden erlebten Räume und Orte von Frieden und Konflikten zentral für die Veränderung der Einstellung. Das AIES bedient sich hier verschiedener Instrumente, die zur Veränderung der Wahrnehmung beitragen und dabei allesamt ausgeprägte räumliche Bezüge haben: die Isolation von der Außenwelt in der Wüste, die Schaffung von gesonderten Räumen für den Austausch über den Konflikt, der Besuch besonderer Schauplätze oder auch die guten Möglichkeiten, als Alumni sowohl physisch als auch virtuell in Kontakt zu bleiben. Gerade der Fortbestand der Maßnahme und deren zeitliche Verlängerung erscheinen bei dem starken Einfluss von alltäglicher Gewalterfahrung auf allen Ebenen der Studierenden entscheidend, um anhaltenden Erfolg im „Frieden machen“ bzw. genauer „Machen von Räumen des Friedens" zu erzielen.

Sowohl die Umwelt als Katalysator als auch die spezifischen Friedensbildungsmaßnahmen führen auf Basis der empirischen Ergebnisse zu partiellen Veränderungen der Konfliktwahrnehmung. Während durch das entstandene soziale Kapital innerhalb der Gruppe eine starke eigene Gruppenidentität entsteht, die auch insgesamt zu einer anderen Rezeption der Konfliktparteien und einem gewissen Aufbruch der Kategorien „wir“ und „die“ führt, hinken Veränderungen in der Perspektive auf das Erbe der Vergangenheit hinterher. Trotz des Willens, in Zukunft und Gegenwart einen Weg der Veränderung im Hinblick auf Frieden und Kompromisse in territorialen Fragen zu gehen, verhaften viele in ihrer Version der Geschichte. Im Vergleich dazu erscheint eine Veränderung der Formulierung von aktuellen territorialen Ansprüchen als einfaches Unterfangen. Einher mit diesem Bruch geht das Spannungsfeld zwischen dem Wunsch nach Frieden in Gegenwart und Zukunft und dem gegenseitigen Anerkennen der erlittenen Traumata; gleichwohl wird auf Basis des erfolgreichen bondings die Bedeutung der $\mathrm{Zu}-$ sammenarbeit der Parteien unterstrichen.

Letztlich zeigt sich wie im eingangs aufgeführten Zitat die Grenze der zivilgesellschaftlichen Konflikttransformation in Projekten wie dem AIES. Für Ausstrahleffekte im Sinne des bridging sowie die Umsetzung der veränderten Wahrnehmung in konkrete Handlungen als Agenten eines positiven Friedens lassen sich einzelne Erfolgsgeschichten formulieren. Die Mehrzahl der Interviewpartner wird jedoch von ihrem Alltag eingeholt und nur durch die Alumniaktivitäten in die Räume des Friedens zurückgeholt; hier ist wiederum der Katalysator Umwelt bedeutend, da auch Alumniaktivitäten und Multiplikatoren in Form von weiterführenden NGOs den Umweltbezug aufgreifen. An dieser Stelle wird die Notwendigkeit von Multi-Track Strategien sichtbar, da die alltäglich erfahrene Gewalt die Effekte des bridging konterkariert. Gleichwohl zeigen sich die Chancen der auf dem Katalysator Umwelt basierenden Konflikttransformation auf Graswurzelebene; vor allem die Wirkungen mit einem größeren Zeithorizont und das Verhalten von Alumni in Entscheidungspositionen in der Zukunft sind hier weitere zu untersuchende Fragen.

Danksagung. Die Autoren möchten sich an dieser Stelle nochmals herzlich bei allen Gesprächspartnern und besonders dem Direktorium des Arava Institute for Environmental Studies für ihre Auskunftsbereitschaft und die Unterstützung unserer Forschungsarbeiten bedanken, daneben beim Editor und den beiden anonymen Gutachtern für ihre ausgesprochen konstruktiven Hinweise. Für den Inhalt des vorliegenden Beitrags bleiben sie selbstverständlich allein verantwortlich.

Edited by: B. Korf

Reviewed by: two anonymous referees

\section{Literatur}

Ali, S. H.: Introduction: A Natural Connection between Ecology and Peace?, in: Peace Parks: Conservation and Conflict Resolution, Herausgeber: Ali, S. H., Massachusetts Institute of Technology, Cambridge, 1-18, 2007.

Bekerman, Z.: Coexistance Education, Encyclopedia of Peace Psychology, Blackwell, 2011.

Bastien, B., Kremer, J., und Vickers, P.: Healing the Impact of colonization, genocide, missionization, and racism on indigenous 
populations, in: The Psychological Impact of War Trauma on Civilians: An International Perspective, Herausgeber: Krippner, S. und McIntyre, T. M., Praeger, Westport, 25-38, 2003.

Bohle, H.-G.: Geographien von Gewalt. Kulturgeographische Interpretationen des Bürgerkrieges auf Sri Lanka, Petermanns Geographische Mitteilungen, 148, 22-29, 2004.

Brown, R.: Beziehung zwischen Gruppen, in: Sozialpsychologie, Herausgeber: Brown, R., Stroebe, W., Jonas, K. und Hewstone, M., Springer, Berlin, 537-576, 2002.

Buckley-Zistel, S. und Moltmann, B.: Versöhnung: Gratwanderung zwischen Wahrheit und Gerechtigkeit, in: Friedensgutachten 2006, Herausgeber: Mutz, R., Schoch, B., Hauswedell, C., Hippler, J. und Ratsch, U., LIT-Verlag, Münster, 168-176, 2006.

Bush, K. und Saltarelli, D.: The two faces of education in ethnic conflict. Towards a Peacebuilding Education for Children, UNICEF Innocenti Research Centre, Florenz, 2000.

Butz, D., und Eyles, J.: Reconceptualizing senses of place: Social relations, ideology and ecology, Geografiska Annaler: Series B, Human Geography, 79, 1-25, 1997.

Castree, N.: David Harvey: Marxism, capitalism and the geographical imagination, New Polit. Econ., 12, 97-115, 2007.

Collier, P.: Breaking the Conflict Trap. Civil War and Development Policy, A World Bank Policy Research Report, Oxford und Washington, 2004

Conca, K. und Dabelko, G. D.: Environmental Peacemaking, Woodrow Wilson Center Press und Johns Hopkins University Press, Washington und Baltimore, 2002.

Conca, K. und Wallace, J.: Environment and peacebuilding in wartorn societies: Lessons from the UN Environment Programme's experience with post-conflict assessment, in: Assessing and Restoring Natural Resources in Post-Conflict Peacebuilding, Herausgeber: Jensen, D. und Lonergan, S., Earthscan, London, 6384, 2012.

Cox, K.: Political geography: territory, state and society, John Wiley, New York, 2008.

Dabelko, G. D. und Rogers, W.: Military-to-Military Cooperation on Environment and Natural Disasters: Engagement for Peacebuilding, in: Governance, Natural Resources, and Post-Conflict Peacebuilding, Herausgeber: Bruch, C., Muffett, C. und Nichols, S. S., Earthscan, London, 2014.

Dabelko, G. D.: An Uncommon Peace: Environment, Development, and the Global Security Agenda, Environment, 50, 32-45, 2008.

Davis, P.: Ecomuseums: A Sense of Place, Leicester University Press, New York, 1999.

Davies, L.: The Different Faces of Education in Conflict, Development, 53, 491-497, 2010.

Doevenspeck, M.: Constructing the border from below: Narratives from the Congolese-Rwandan state boundary, Polit. Geogr., 30, 129-142, 2011.

Dollase, R.: Fremdenfeindlichkeit verschwindet im Kontakt von Mensch zu Mensch. Zur Reichweite der Kontakthypothese, Diskurs, 11, 16-21, 2001.

Floyd, R.: Whither environmental security studies? An afterword, in: Environmental security: approaches and issues, Herausgeber: Floyd, R. und Matthew, R., Routledge, London, 279-297, 2013.

Fukuyama, F.: State-building: governance and world order in the 21 st century, Cornell University Press, 2004.
Galtung, J.: Peace by Peaceful Means: Peace and Conflict, Development and Civilization, International Peace Research Institute, London, 1996.

Gregory, D.: The Colonial Present: Afghanistan, Palestine and Iraq, Blackwell, Malden, 2004.

Houdret, A.: Wasserkonflikte sind Machtkonflikte. Ursachen und Lösungsansätze in Marokko, Wiesbaden, 2010.

Hurley, J. M., Ginger, C., und Capen, D. E.: Property Concepts, Ecological Thought, and Ecosystem Management: A Case of Conservation Policymaking in Vermont, Society and Natural Resources, 15, 295-312, 2002.

Ingold, T.: The perception of the environment: essays on livelihood, dwelling and skill, Routledge, New York, 2000.

Jäger, U.: Multi-Track: Anregungen zur Konzeption einer zeitgemäBen Friedenspädagogik, ZEP: Zeitschrift für internationale Bildungsforschung und Entwicklungspädagogik, 33, 28-32, 2010.

Jonas, K.: Die Kontakthypothese: Abbau von Vorurteilen durch Kontakt mit Fremden, Die offene Gesellschaft und ihre Fremden, 129-154, 1998.

Korf, B. und Engler, M.: Geographien der Gewalt, Zeitschrift für Wirtschaftsgeographie, 51, 221-237, 2007.

Korf, B.: Klimakriege: Zur Politischen Ökologie der „Kriege der Zukunft“, Handbuch Kriegstheorien, 577-585, 2012.

Korf, B. und Raeymaekers, T.: Geographie der Gewalt, Geographische Rundschau, 64, 4-11, 2012.

Korf, B. und Schetter, C.: Geographien der Gewalt, Kriege, Konflikte und die Ordnung des Raumes im 21. Jahrhundert, Borntraeger, 2015.

Kyrou, C. N.: Peace Ecology: An Emerging Paradigm in Peace Studies, International Journal of Peace Studies, 12, 73-99, 2007.

Lederach, J. P.: Conflict Transformation in Protracted Internal Conflicts: The Case for a Comprehensive Framework, in: Conflict Transformation, Herausgeber: Rupesinghe, K., Houndsmills, Basingstoke, Hampshire und London, 201-222, 1995.

Lederach, J. P.: Building Peace: Sustainable Reconciliation in Divided Societies, United States Institute of Peace Press, Washington, 1997.

Lenhart, V., Mitschke, R., und Braun, S.: Friedensbauende Bildungsmaßnahmen bei bewaffneten Konflikten. Zwischenergebnisse eines Forschungs-und Entwicklungsprojektes, ZEP: Zeitschrift für internationale Bildungsforschung und Entwicklungspädagogik, 30, 17-22, 2007.

Marten, K. Z.: Enforcing the peace: Learning from the imperial past, Columbia University Press, 2004.

Putnam, R.: Bowling alone: The collapse and revival of community in America, Simon and Schuster, New York, 2000.

Reuber, P. und Strüver, A.: Diskursive Verräumlichungen in deutschen Printmedien: Das Beispiel Geopolitik nach 9/11, in: Mediengeographie. Theorie - Analyse - Diskussion, Herausgeber: Döring, J. und Thielmann, T., Transcript, Bielefeld, 315-332, 2009.

Rodrian, P.: Heritage als Konstruktion touristischer Mediatoren: Die Darstellung des deutschen Kolonialerbes in Namibia durch Print-Reiseführer und Reiseleiter, Zeitschrift für Tourismuswissenschaft, 3, 25-41, 2011. 
Rodrian, P., Fricke, N. und Zademach, H.-M.: Großschutzgebiete als Instrument der Konflikttransformation: Betrachtungen im Licht der Peace Ecology, in: Naturschutz in geographischer Perspektive, Herausgeber: Erdmann, K.-H., Bork H.-R. und Job, H., BfN-Skripten 400, Bonn, 123-136, 2015.

Ruschkowski, E. v. und Mayer, M.: From Conflict to Partnership? Interactions between Protected Areas, Local Communities and Operators of Tourism Enterprises in Two German National Park Regions, Journal of Tourism and Leisure Studies, 17, 147-181, 2011.

Rothfuß, R.: Geographische Konfliktforschung und Geopolitik: Zukunftsaufgabe Friedenssicherung, Jahresheft Geopolitik, 4, 3645, 2011.

Salomon, G.: Lessons from Research on Peace Education in Israel/Palestine, Asian Journal of Peacebuilding, 1, 1-15, 2013.

Salomon, G. und Cairns, E.: Peace Education. Setting the Scene, in: Handbook on Peace Education, Herausgeber: Salomon, G. und Cairns, E., Taylor \& Francis, New York, 2010.

Salomon, G.: Peace Education: Its Nature, Nurture and the Challenges It Faces, in: Handbook on Building Cultures of Peace, Herausgeber: de Rivera, J., Peace Psychology Book Series, Springer, New York, 2009.

Smith, A.: The influence of education on conflict and peace building, in: UNESCO: Paper commissioned for the EFA Global Monitoring Report 2011. The hidden crisis: Armed conflict and education, 2010.
Stürmer, S.: Die Kontakthypothese, in: Stereotype, Vorurteile und soziale Diskriminierung: Theorien, Befunde und Interventionen, Herausgeber: Petersen, L.-E. und Six, B., Beltz, Weinheim, 283291, 2008.

Springer, S.: Violence sits in places. Cultural practice, neoliberal rationalism, and virulent imaginative geographies, Political Geography, 30, 90-98, 2011.

Van Gielle Ruppe, P., Helbrecht, I. und Dirksmeier, P.: Die Politisierung der Stadtplanung: die performative Rolle von Planungsinstrumenten in Konfliktzonen am Beispiel Jerusalem, Raumforschung und Raumordnung, 70, 411-424, 2012.

Vickers, V. G. und Matthews, C. E.: Children and Place: A Natural Connection, Science Activities: Classroom Projects and Curriculum Ideas, 39, 16-24, 2002.

Vukosavljevic, N.: Training for Peacebuilding and Conflict Transformation. Experiences of the "Centre for Nonviolent Action" in the Western Balkans, www.berghof-handbook.net, Berghof Forschungszentrum für konstruktive Konfliktbearbeitung, 2007.

Watts, M.: Geographies of violence and the narcissism of minor difference, Struggles over geography: Violence, freedom and development at the millennium, Hettner lecture, 3, Heidelberg, 7-34, 2000.

Wehr, P.: Conflict Regulation, Westview Press, Boulder, 1979.

Wilmot, W. und Hocker, J.: Interpersonal Conflict, McGraw-Hill, New York, 2001. 